\title{
On solitary waves forced by underwater moving objects
}

\author{
By DAOHUA ZHANG and ALLEN T. CHWANG \\ Department of Mechanical Engineering, The University of Hong Kong, \\ Pokfulam Road, Hong Kong
}

(Received 20 May 1997 and in revised form 28 December 1998)

The phenomenon of a succession of upstream-advancing solitary waves generated by underwater disturbances moving steadily with a transcritical velocity in twodimensional shallow water channels is investigated. The two-dimensional NavierStokes (NS) equations with the complete set of viscous boundary conditions are solved numerically by the finite-difference method to simulate the phenomenon. The overall features of the phenomenon illustrated by the present numerical results are unanimous with observations in nature as well as in laboratories. The relations between amplitude and celerity, and between amplitude and period of generation of solitary waves can be accurately simulated by the present numerical method, and are in good agreement with predictions of theoretical formulae. The dependence of solitary wave radiation on the blockage and on the body shape is investigated. It furnishes collateral evidence of the experimental findings that the blockage plays a key role in the generation of solitary waves. The amplitude increases while the period of generation decreases as the blockage coefficient increases. It is found that in a viscous flow the shape of an underwater object has a significant effect on the generation of solitary waves owing to the viscous effect in the boundary layer. If a change in body shape results in increasing the region of the viscous boundary layer, it enhances the viscous effect and so does the disturbance forcing; therefore the amplitudes of solitary waves increase. In addition, detailed information of the flow, such as the pressure distribution, velocity and vorticity fields, are given by the present NS solutions.

\section{Introduction}

After the discovery of solitary waves by John Scott Russell, it is now well known that a forcing disturbance moving steadily at a transcritical velocity on or beneath the free surface of a shallow water channel can generate, periodically, a succession of upstream-advancing solitary waves, while a region of ever elongating depressed water surface develops immediately behind the disturbance, followed by a train of cnoidal-like waves gradually attenuating in the far field. The salient feature of this phenomenon is that the motion of the disturbance is steady but the flow excited does not attain a steady state. The first experimental study of this phenomenon was in a report of towing tank tests of ship models in shallow water by Thews \& Landweber (1935). Thereafter, laboratory studies on this phenomenon were performed by several investigators by moving vessels in comparatively narrow channels (Graff 1962; Schmidt-Stiebitz 1966; Huang et al. 1982; Ertekin, Webster \& Wehausen 1984; Sun 1985). 
This phenomenon has been the subject of many theoretical studies. $\mathrm{Wu} \& \mathrm{Wu}$ (1982) provided theoretical evidence for the excitation of solitary waves near critical conditions for the case of plane motions by numerically solving the generalized Boussinesq ( $\mathrm{g}$-B) equation using a surface pressure distribution or a bottom topography as forcing functions. Calculations using a surface pressure or bottom disturbance as a forcing term were performed by Akylas (1984) and Cole (1985) based on the Korteweg-de Vries (KdV) equation, yielding qualitatively similar results. In a joint theoretical and experimental study, Lee, Yates \& Wu (1989) found a broad agreement between experiments and two theoretical models, i.e. the g-B and the forced Korteweg-de Vries (fKdV) equation. Wu (1987) carried out a preliminary study to ascertain the mechanism underlying the phenomenon by approximately analysing the $\mathrm{fKdV}$ equation. For three-dimensional disturbances, such as a real ship, Mei (1986) derived an inhomogeneous $\mathrm{KdV}$ equation with matched asymptotic expansions to analyse a slender ship hull. This theory was further developed and improved by Mei \& Choi (1987), and Chen \& Sharma $(1994,1995)$. The problem of a three-dimensional surface-pressure distribution used to simulate a ship hull moving in a channel was studied numerically by Ertekin, Webster \& Wehausen (1986) based on Green-Naghdi's directed-sheet model, by Katsis \& Akylas (1987) using the Kadomtsev-Petviashvili equation, and by Pedersen (1988) based on the Boussinesq-type equations.

Besides the simplified theoretical studies of this phenomenon, many numerical schemes for potential flows with fully nonlinear boundary conditions on the free surface have been presented. Bai \& Kim (1989) presented a finite-element method based on the Laplace equation for solving a nonlinear free-surface flow for a simple wedge-shaped ship moving in a towing tank. Protopopov (1991) developed a finitedifference method to study solitary waves generated by a two-dimensional pressure distribution moving on the free surface. Choi et al. (1991) studied the generation of solitary waves by a three-dimensional ship moving in a channel using a finite-element method. Cao \& Beck (1993) presented a simple numerical method to compute the solitary waves generated by three types of disturbance, i.e. a pressure distribution on the free surface, a bottom topography, and a submerged cylinder, using the Laplace equation and fully nonlinear boundary conditions.

The theoretical and numerical studies of the phenomenon mentioned above are based on the potential-flow theory. The main drawback of these methods is that the viscous effects are either neglected, or taken into account in a very crude way. To analyse the complexities of the phenomenon including the viscous effects, a more general model for solving time-dependent viscous flow with a free surface is required. Solutions of NS equations with consistent viscous free-surface boundary conditions offer an opportunity to capture these complexities. Methods of this type have been developed for a variety of water-wave problems in hydrodynamics (Harlow \& Welch 1965; Chan \& Street 1970; Miyata \& Nishimura 1985; Yeung \& Vaidhyanathan 1992). Chang \& Tang (1993) studied solitary waves generated by a bottom bump based on the vorticity-stream function formulation by means of the finite-analytic method, and found that the amplitudes of advancing solitary waves and downstream trailing waves were attenuated by the viscous effect at low Reynolds numbers. Zhang \& Chwang (1996) carried out a comprehensive investigation on solitary waves produced by submerged moving objects. Their numerical solutions of the NS equations were in excellent agreement with the experimental data of Lee et al. (1989). The investigation revealed that the source of vorticity at the bottom of the channel and in the boundary layer of a submerged body is much stronger than that on the free surface. Although viscous action on the free surface may have some effect, 
the viscous effect in the boundary layer around the body, and on the floor of the channel, plays an important role in the generation of solitary waves. The excellent agreement with experiments by the numerical solutions of NS equations is primarily due to the fact that the viscous effects in the boundary layer around the body and on the floor of the channel can be correctly simulated in the computation of the NS formulation. The investigation showed that waves generated are insensitive to the submergence depth of the body, except when the body is quite close to the bottom of the channel. This supports the theory and agrees with the experimental observation.

The present work can be seen as a sequel to the previous study (Zhang \& Chwang 1996) and is primarily concerned with the dependence of solitary-wave radiation on the shape of the submerged transcritically moving object and on the blockage coefficient. In the experimental study of Ertekin et al. (1984), it was pointed out that the dominant factor governing the generation of solitary waves is the blockage coefficient, defined as the ratio of the mid-ship-sectional area to the cross-sectional area of the channel. Based on the existing inviscid results, different studies (e.g. Ertekin et al. 1984, 1986; Mei 1986; Ertekin \& Qian 1986; Teng \& Wu 1997) have shown that if the disturbance length is sufficiently long (much larger than the water depth), the dominant forcing parameter is the blockage coefficient, while the shape of the disturbance does not have a significant effect. When the disturbance length is of the same order as the water depth, however, the shape of the disturbance will have a significant effect on the waves. Thus, the objective of the present study is to verify the blockage coefficient concept and to examine numerically the effect of disturbance shape on the generation of solitary waves. The important relations among key flow quantities, such as the relations between amplitude and celerity, and between amplitude and period of generation, are investigated. The numerical method employed for calculations is outlined in $\S 2$. Calculations are performed for a series of elliptic cylinders of the same volume (the same cross-sectional area in the present two-dimensional case) but of different major-to-minor axis ratios, moving at a transcritical velocity in a channel of different water depths resulting in different blockage coefficients. The results of these calculations are presented in $\S 3$, where the general characteristics of the solitary waves generated are described; the detailed information of the flow is presented; and the effects of the blockage coefficient and the shape of submerged bodies on the generation of solitary waves are examined. Finally, conclusions are given in $\S 4$.

\section{Outline of the numerical method}

The present computational method is based on the approach of Zhang \& Chwang (1996). Only an outline of the method is given in this section. A Cartesian coordinate system $\left(x_{i}\right)$ is established as the reference frame, with the origin fixed on the mean free surface at the mid-body, the $x_{1}$-axis in the downstream direction and the $x_{2}$ axis pointing vertically upwards (see figure 1). The motion of an incompressible viscous fluid under the influence of gravity is governed by the Navier-Stokes (NS) equations and the continuity equation, which in a boundary fitted coordinate system $\left(\xi^{j}\right), \xi^{j}=\xi^{j}\left(x_{i}, t\right)$, are given by

$$
\frac{\partial}{\partial t}\left(\frac{u_{i}}{J}\right)+\frac{\partial}{\partial \xi^{j}}\left(\frac{u_{i}}{J} \frac{\partial \xi^{j}}{\partial t}+\frac{u_{i}}{J} U^{j}\right)=-\frac{\partial}{\partial \xi^{j}}\left(\frac{\phi}{J} \frac{\partial \xi^{j}}{\partial x_{i}}\right)+\frac{1}{R e} \frac{\partial}{\partial \xi^{j}}\left(\frac{1}{J} g^{j l} \frac{\partial u_{i}}{\partial \xi^{l}}\right),
$$




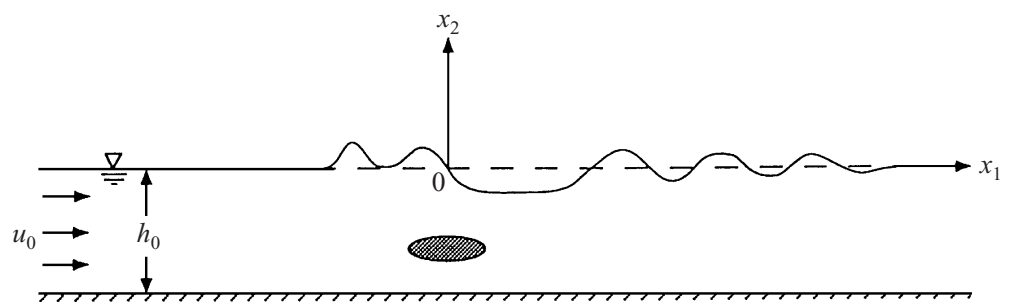

FIGURE 1. Schematic diagram of an obstacle fixed in a uniform stream of velocity $u_{0}$.

and

with

$$
\frac{\partial}{\partial \xi^{j}}\left(\frac{U^{j}}{J}\right)=0
$$

$$
J=\frac{\partial\left(\xi^{1}, \xi^{2}\right)}{\partial\left(x_{1}, x_{2}\right)}, \quad U^{j}=\frac{\partial \xi^{j}}{\partial x_{k}} u_{k}, \quad \phi=p+\frac{x_{2}}{F n^{2}}, \quad g^{j l}=\frac{\partial \xi^{j}}{\partial x_{k}} \frac{\partial \xi^{l}}{\partial x_{k}},
$$

where $u_{i}$ is the velocity component along the $x_{i}$-axis, $t$ is the time, $J$ is the Jacobian of the transformation, $U^{j}$ is the contravariant velocity component along the $\xi^{j}$ direction, $g^{j l}$ is the metric tensor, $\phi$ is the redefined pressure term, $p$ is the pressure, and $\partial \xi^{j} / \partial t$ associated with the grid movement is the grid point speed. The governing equations are normalized by the uniform stream velocity (or in a laboratory frame, the steady velocity of the disturbance) $u_{\mathrm{o}}$ and the initial uniform water depth $h_{\mathrm{o}}$. The Reynolds and Froude numbers are defined as $R e=u_{0} h_{0} / v$ and $F n=u_{0} /\left(g h_{0}\right)^{1 / 2}$, respectively, where $g$ is the gravitational constant and $v$ is the kinematic viscosity coefficient.

The free-surface boundary is not known a priori. The location of the free surface is part of the solution in the problem and is determined from the free-surface boundary conditions which consist of one kinematic condition and two dynamic conditions. The kinematic condition, which states that fluid particles of the free surface stay on the free surface at any time, is

$$
\frac{\partial h}{\partial t}+\left(\frac{\partial \xi^{i}}{\partial t}+u_{1} \frac{\partial \xi^{i}}{\partial x_{1}}\right) \frac{\partial h}{\partial \xi^{i}}=u_{2} \quad \text { on the free surface. }
$$

The dynamic conditions represent the continuity of stresses on the free surface. Neglecting the surface tension on the free surface, the dynamic conditions are

$$
n_{i} \sigma_{i j} n_{j}=0
$$

and

$$
t_{i} \sigma_{i j} n_{j}=0 \text { on the free surface, }
$$

where $n_{i}$ is the unit outward normal vector and $t_{i}$ the unit tangential vector to the free surface. The stress tensor for an incompressible Newtonian fluid can be expressed as

$$
\sigma_{i j}=-p \delta_{i j}+\frac{1}{R e}\left(\frac{\partial u_{j}}{\partial \xi^{l}} \frac{\partial \xi^{l}}{\partial x_{i}}+\frac{\partial u_{i}}{\partial \xi^{l}} \frac{\partial \xi^{l}}{\partial x_{j}}\right)
$$

where $\delta_{i j}$ is the Kronecker delta.

The no-slip boundary condition is imposed on the body surface and on the bottom of the channel. The solution domain is made sufficiently large in the upstream direction so that the generated solitary waves travelling upstream do not hit the upstream boundary at the final time of computation. Thus, at the far upstream, flow 
is uniform and the free surface is undisturbed. However, the downstream boundary condition must be carefully implemented to prevent the reflection of waves into the solution domain. The added dissipation zone method of Chiba \& Kuwahara (see Hinatsu 1992) is used for the downstream boundary. The initial velocity field is taken to be that of the static (quiescent) fluid case, and a constant acceleration is imposed in the entire domain until the inflow velocity attains one.

A regular grid is used in the present method for discretization. The velocity and pressure are evaluated at computational cell centres. Spatial differentials are discretized using the second-order central difference, and the QUICK scheme of Leonard (1979) is used for discretization of convection terms. Time marching is carried out using a time-splitting fractional-step method. It is a two-step predictorcorrector scheme. In the predictor step, an intermediate velocity field $\tilde{u}_{i}$ is computed explicitly by integrating equation (1) in time, using the velocity and pressure from previous timestep $n$,

$$
\frac{\tilde{u}_{i}}{J^{n+1}}-\left(\frac{u_{i}}{J}\right)^{n}=\Delta t\left[-\frac{\partial}{\partial \xi^{j}}\left(\frac{u_{i}}{J} U^{j}\right)-\frac{\partial}{\partial \xi^{j}}\left(\frac{\phi}{J} \frac{\partial \xi^{j}}{\partial x_{i}}\right)+\frac{1}{R e} \frac{\partial}{\partial \xi^{j}}\left(\frac{1}{J} g^{j l} \frac{\partial u_{i}}{\partial \xi^{l}}\right)\right]^{n},
$$

where $\Delta t$ is the time increment and the superscripts $n$ and $n+1$ denote the previous and the present timesteps, respectively. After the predictor step, the location of the free surface at timestep $n+1$ is evaluated by integrating equation (4) explicitly,

$$
h^{n+1}=h^{n}+\Delta t\left(u_{2}-u_{1} \frac{\partial \xi^{i}}{\partial x_{1}} \frac{\partial h}{\partial \xi^{i}}\right)^{n},
$$

and the grid of the flow domain is regenerated to fit the newly estimated free surface. The Jacobian and the metric tensor are recalculated according to the new grid.

At the second step, $\tilde{u}_{i}$ is corrected by the pressure increment $\delta \phi=\phi^{n+1}-\phi^{n}$ between the present timestep $n+1$ and the previous timestep $n$ to obtain the velocity field $u_{i}^{n+1}$ at the present timestep $n+1$

$$
\begin{aligned}
\left(\frac{u_{i}}{J}\right)^{n+1}-\frac{\tilde{u}_{i}}{J^{n+1}} & =-\Delta t\left[\frac{\partial}{\partial \xi^{j}}\left(\frac{\phi}{J} \frac{\partial \xi^{j}}{\partial x_{i}}\right)\right]^{n+1}+\Delta t\left[\frac{\partial}{\partial \xi^{j}}\left(\frac{\phi}{J} \frac{\partial \xi^{j}}{\partial x_{i}}\right)\right]^{n} \\
& \cong-\Delta t\left[\frac{\partial}{\partial \xi^{j}}\left(\frac{\delta \phi}{J} \frac{\partial \xi^{j}}{\partial x_{i}}\right)\right]^{n+1} .
\end{aligned}
$$

Note that the difference of the pressure differential between $n+1$ and $n$ timesteps is simply approximated by the differential of the pressure increment at $n+1$ timesteps. However, the pressure increment $\delta \phi$ in equation (10) is not known and must be determined by requiring the velocity field $u_{i}^{n+1}$ to satisfy the continuity equation. The divergence-free condition applied to $u_{i}^{n+1}$ yields a Poisson equation for $\delta \phi$ by taking the divergence of equation (10). Thus,

$$
\left[\frac{\partial}{\partial \xi^{i}}\left(\frac{\tilde{U}^{i}}{J}\right)\right]^{n+1}=\Delta t\left\{\frac{\partial}{\partial \xi^{k}}\left[\frac{\partial \xi^{k}}{\partial x_{i}} \frac{\partial}{\partial \xi^{j}}\left(\frac{\delta \phi}{J} \frac{\partial \xi^{j}}{\partial x_{i}}\right)\right]\right\}^{n+1}
$$

where $\tilde{U}^{i}=\left(\partial \xi^{i} / \partial x_{j}\right) \tilde{u}_{j}$. The pressure at timestep $n+1$ is then obtained by $\phi^{n+1}=$ $\phi^{n}+\delta \phi$.

The unsteady problem is treated as a quasi-steady one between successive physical 


\begin{tabular}{ccccc}
\hline $\begin{array}{c}\text { Case } \\
\text { number }\end{array}$ & $\begin{array}{c}\text { Major-minor } \\
\text { axis ratio }(a / b)\end{array}$ & $\begin{array}{c}\text { Water depth } \\
h_{0}(\mathrm{~cm})\end{array}$ & $\begin{array}{c}\text { Blockage coefficient } \\
\left(C_{b}=2 b / h_{0}\right)\end{array}$ & $\begin{array}{c}\text { Reynolds } \\
\text { number }(R e)\end{array}$ \\
1 & 6 & 4.00 & 0.200 & $3.0068 \times 10^{4}$ \\
2 & 6 & 4.57 & 0.175 & $3.2129 \times 10^{4}$ \\
3 & 6 & 5.33 & 0.150 & $3.4687 \times 10^{4}$ \\
4 & 6 & 6.40 & 0.125 & $3.8033 \times 10^{4}$ \\
5 & 6 & 8.00 & 0.100 & $4.2523 \times 10^{4}$ \\
6 & 2 & 6.91 & 0.200 & $2.2757 \times 10^{4}$ \\
7 & 4 & 4.89 & 0.200 & $2.7095 \times 10^{4}$ \\
8 & 8 & 3.45 & 0.200 & $3.2113 \times 10^{4}$ \\
9 & 10 & 3.09 & 0.200 & $3.4025 \times 10^{4}$
\end{tabular}

TABLE 1. Parameters used for the nine elliptic cylinders.

timesteps. The known value of a dependent variable at time $t$ can be used as an initial guess for the unknown value of that variable at time $t+\Delta t$ for reasonably small $\Delta t$. Since this is a relatively good guess, only a few iterations are normally needed to obtain a convergent solution for time $t+\Delta t$. The grid is aligned to the free-surface boundary, which moves in time. Therefore, the grid is time dependent in general. However, equation (1) is integrated in time by the explicit method, within each timestep the grid is independent of time (see Farmer, Martinelli \& Jameson 1994). In an explicit method, which must use small timesteps, the problem associated with grid movement is often ignored (see Ferziger \& Peric 1996). Therefore, the grid-point speed term, $\partial \xi^{j} / \partial t$, is dropped in equations (8) and (9).

\section{Discussion of results}

In the experimental study of Ertekin et al. (1984), it was pointed out that the dominant parameter governing the generation of solitary waves is the blockage coefficient, defined as the ratio of the mid-ship-sectional area to the cross-sectional area of the channel. Zhang \& Chwang (1996) showed that in experimental situations (the Reynolds numbers are in the range of $10^{4}$ ) the viscous effect in the boundary layer around a body and on the floor of a channel plays an important role in the generation of solitary waves, indicating that the body shape has a significant effect on the properties of solitary waves generated. Therefore, a systematic study is carried out to investigate the effect of the blockage and the effect of the body shape on the generation of solitary waves. It is difficult to define accurately the shape of an arbitrary body and to account for the effect of trivially changing its shape on waves generated. Therefore, elliptic cylinders of the same cross-sectional area but different aspect ratios are chosen for the investigation of the shape effect. Nine elliptic cylinders in laboratory scale, all having the same cross-sectional area of $3 \mathrm{~cm}^{2}$, are used for calculations to investigate the effects of blockage and shape on the waves generated. The parameters used for the nine calculation cases are listed in table 1. The Reynolds numbers are based on the body length along the uniform flow direction and the Froude numbers are all equal to one. The calculations can be organized into two groups: group 1 (cases 1 to 5) for investigating the effect of blockage coefficient on solitary-wave generation, and group 2 (cases 6, 7, 1, 8, and 9) for studying the shape effect on solitary-wave generation.

The calculation domains for all the cases are the same, i.e. 120 units in the horizontal 
direction and 1 unit in the vertical direction. Two-hundred and seventy grid points are used in the horizontal direction and 47 grid points are given in the vertical direction. The grid lines are clustered to the solid wall of the body, to the bottom of the channel, and to the free surface with a minimum grid spacing of 0.002 adjacent to the wall in the vertical direction (see figure 4). The centroid depth of the elliptic cylinders is -0.7 . The timestep is set to be $\Delta t=0.01$. The computations start at the initial condition of zero velocity and pressure everywhere and a flat free surface. A constant acceleration of 0.33 , or $0.33 \mathrm{~g}$ in the dimensional space, is imposed on the entire domain until the uniform flow velocity reaches one. For the timestep of $\Delta t=0.01,300$ timesteps are needed to accelerate the uniform flow velocity from zero to one. Two numerical wave gauges are set up in the calculations to record the waves generated. One is fixed with the cylinder and positioned at its leading edge to record the 'birth' of the upstream-advancing waves, and another is located at 70 units upstream of the cylinder initially and then moves with the uniform stream to measure the free-surface elevation as a function of time.

\subsection{General character of the calculated solitary waves}

Before the discussion on the blockage and shape effects on the generation of solitary waves, the properties of upstream-advancing solitary waves calculated by the present numerical method, such as the preiod of generation, the wave speed, and some other pertinent flow quantities, are examined.

The free-surface profiles at various times from the present numerical results of calculation case 1 are shown in figure 2. This plot shows the development of the surface-wave elevation with time as viewed in the body frame. The overall features of the aforementioned phenomenon of solitary waves are clearly shown by the plot. It is evident that waves are generated periodically at the disturbance and developed into waves of almost constant amplitude, propagating upstream without changing shape at a constant velocity $c-u_{o}$ relative to the body, $c$ being the amplitude-dependent phase velocity in the fluid frame. The phase velocity $c$ can be determined with reasonable accuracy by the slope of a straight line connecting the crests of solitary waves at different times in the plot. One of the important properties of solitary waves generated by a disturbance moving at the critical speed is that their speed is supercritical and related to the amplitude. Two theoretical formulae, one being $c=(1+\alpha)^{1 / 2}$ given by Rayleigh, and the other being $c=1+0.5 \alpha$ given by the KdV model (cf. Teng \& $\mathrm{Wu}$ 1992) are available for expressing the relation between the phase velocity $c$ of a solitary wave and its amplitude $\alpha$. The relations between $c$ and $\alpha$ of the leading solitary waves corresponding to all nine cases in table 1 are shown in figure 3 together with those estimated by the two theoretical formulae. It can be seen that the present numerical results lie in between the estimations of the two theoretical formulae. For small $\alpha$, the present results have a better agreement with those given by the Rayleigh formula, whereas for large $\alpha$ the present results are about the average of the values from the two theoretical formulae.

Another important property of solitary waves generated by a transcritically moving object is that the period of solitary-wave generation is related to its amplitude. Wu (1987) derived an approximate formula based on mass, momentum and energy considerations of the $\mathrm{fKdV}$ equation to express the relation between the period of solitary-wave generation $T_{S}$ and wave amplitude $\alpha$ as $T_{S}=12.3 / \alpha^{3 / 2}$. The relation between the period of solitary-wave generation and its amplitude obtained by the present numerical method is in good agreement with Wu's formula, and both show that the period of solitary-wave generation decreases as the wave amplitude increases. 


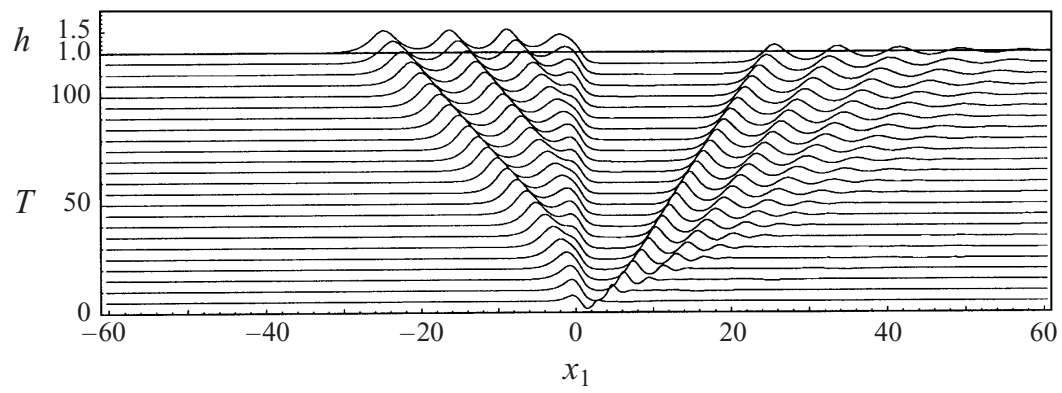

FiguRE 2. Free-surface profiles at various time $t$ for calculation case 1 .

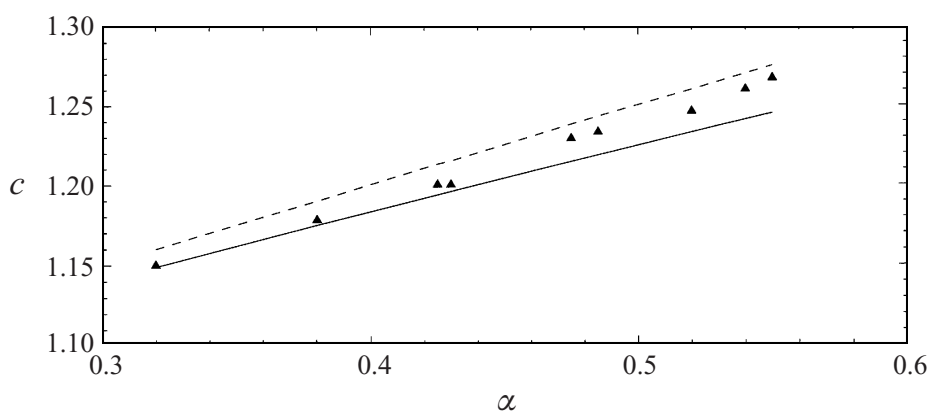

FIGURE 3. Wave speed $c$ versus wave amplitude $\alpha$. $\boldsymbol{\Delta}$, present NS solution;

$$
\longrightarrow,(1+\alpha)^{1 / 2} ;---, 1+\frac{1}{2} \alpha \text {. }
$$

Figures 4 and 5 show the velocity vector plot and vorticity contours for calculation case 1 at time $t=120$, respectively. The viscous thin boundary layers around the cylinder and on the part of the channel floor under the cylinder, and the flow separation at the lower part of the trailing edge are clearly shown in these figures. The presence of the viscous boundary layers may be thought to increase the effective strength of the cylinder, thus affecting the generation of solitary waves. The pressure distribution in the flow for calculation case 1 at time $t=120$ is given in figure 6 . It can be seen that the pressure is positive under the solitary waves, but negative in the depressed region and at the upper part of the trailing edge of the cylinder.

\subsection{The effect of blockage on the generation of solitary waves}

In the experiments of Ertekin et al. (1984), a model of a Series $60, C_{B}=0.80$ ship hull was used, where $C_{B}$ is the Block coefficient (see Newman 1980). By systematically varying the water depth, draft and width of the tank, a wide range of blockage coefficients was obtained. It was found that the amplitude of solitary waves and the time period of generation depend only on the blockage coefficient. When the blockage coefficient is made equal, it would generate forward advancing solitary waves with the same wave elevation and the same period of generation. Based on the slender-body theory, Mei (1986) derived an inhomogeneous KdV equation in which the forcing term is related to the blockage coefficient. Two specific cases with the smallest blockage coefficient corresponding to Ertekin's experiments were simulated. The results agreed well with most of the observations of Ertekin et al. (1984). Later, Ertekin, Qian \& Wehausen (1990) used the g-B equation proposed by Wu (1981) to calculate the precursor solitons generated by a strut of parabolic cross-section. 


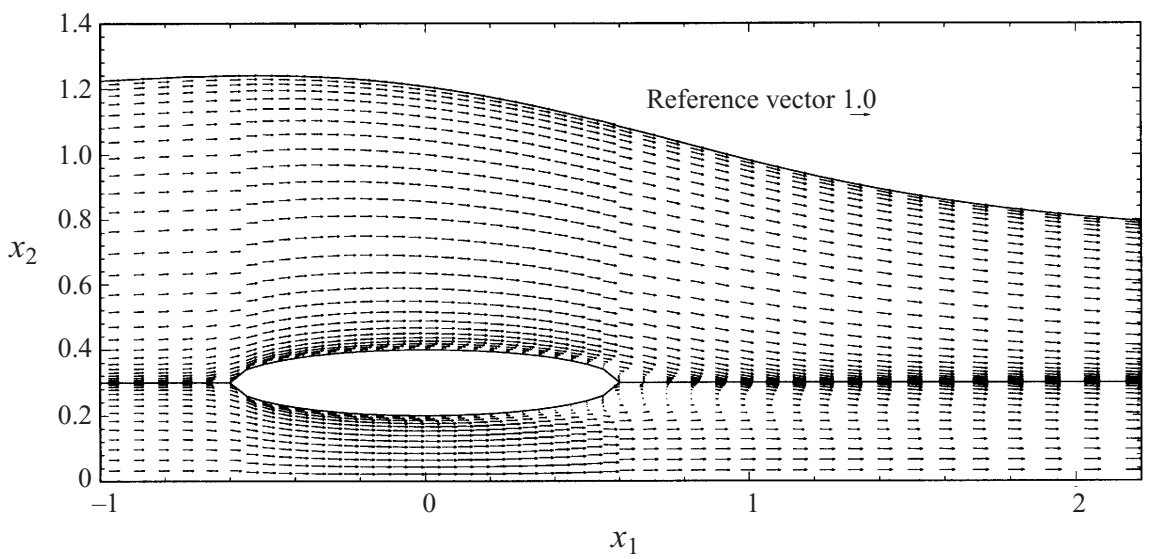

FIGURE 4. Velocity-vector plot at $t=120$ for calculation case 1.

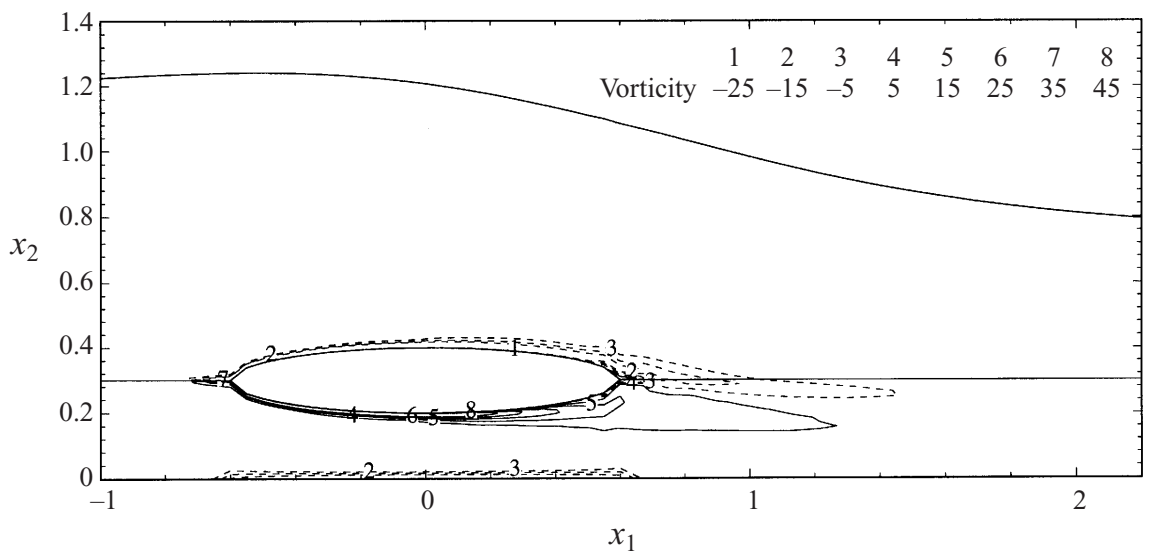

FIGURE 5. Vorticity contours at $t=120$ for calculation case 1 .

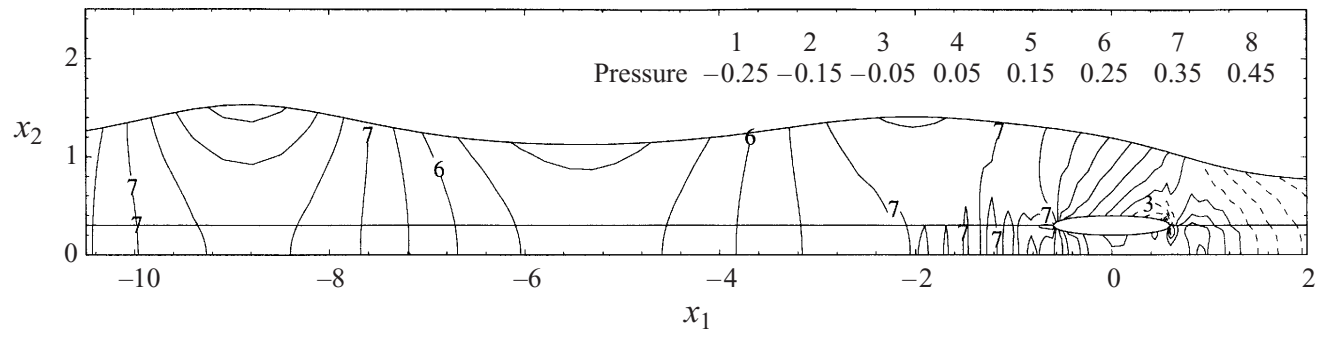

FIgURE 6. Pressure contours at $t=120$ for calculation case 1 .

In order to facilitate comparison with the experiments of Ertekin et al. (1984), the calculations matched the blockage coefficients in various experiments, and the ratios of the half tank width to the water depth. The agreement between the computed values for the strut and the measured values for the ship model indicates that the blockage coefficient is of primary importance in determining the properties of the precursor solitons.

In the present study, numerical simulations based on the NS equations are carried 


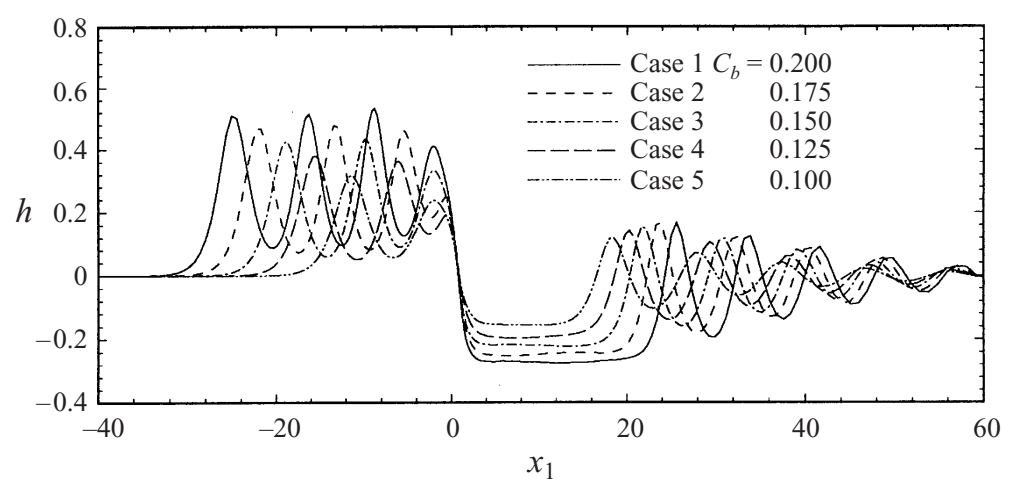

FIGURE 7. Free-surface elevation $h$ at time $t=120$ for cylinders of the same shape $(a / b=6)$ with different blockage coefficient $C_{b}$.

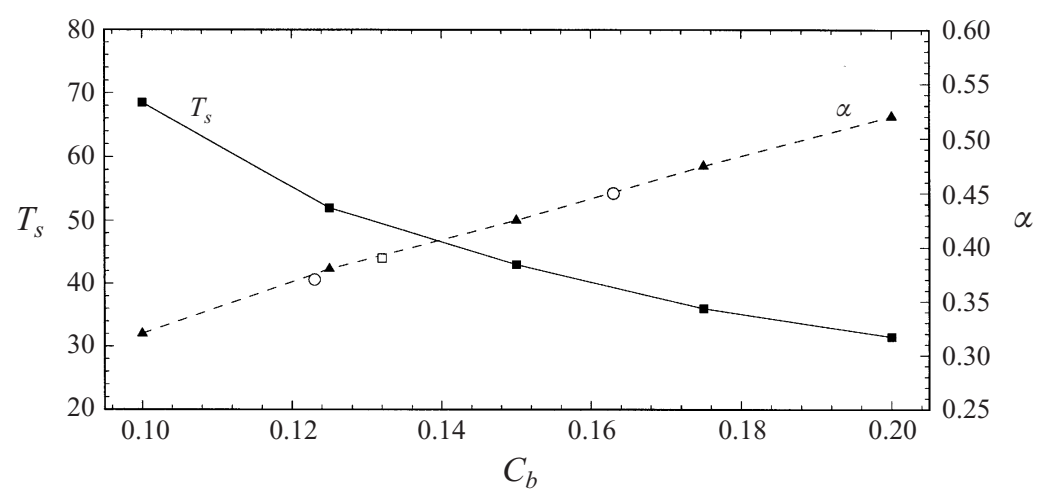

FIGURE 8. Wave amplitude $\alpha$ and period $T_{S}$ versus blockage coefficient $C_{b}$. o, experiment of Lee et al. (1989); $\square$, experiment of Teng \& Wu (1997).

out to investigate the effect of the blockage coefficient on the solitary-wave generation. An elliptic cylinder of $2.4 \mathrm{~cm}$ semi-major axis and $0.4 \mathrm{~cm}$ semi-minor axis is used for the calculations. Five different water depths corresponding to five different blockage coefficients are adopted for calculation cases 1 to 5 in table 1 . Wave elevations at time $t=120$ for all calculation cases are presented in figure 7 . The figure shows that the amplitudes of upstream runaway solitary waves and the downstream trailing waves increase as the blockage coefficient increases. As for the depressed water region immediately behind the moving disturbance, the water depth of the region decreases while its length increases as the blockage coefficient increases. Figure 8 shows the amplitude of the first solitary wave, $\alpha$, and the period of the solitary wave generation, $T_{S}$, versus the blockage coefficient. It can be seen that the amplitude increases while the period decreases as the blockage coefficient increases.

The available experimental data of Lee et al. (1989) and Teng \& Wu (1997) are included in figure 8 for comparison. In the theoretical and experimental study of Lee et al. (1989), a two-dimensional body of an arched cross-section with a chord of $4.9 \mathrm{~cm}$, height of $0.65 \mathrm{~cm}$, and span of $72 \mathrm{~cm}$ was used. Two water depths, $h_{0}=4.0 \mathrm{~cm}$ and $h_{0}=5.33 \mathrm{~cm}$, corresponding to two different blockage coefficients, 0.163 and 0.123 , were used for the study. Numerical results from the $\mathrm{g}-\mathrm{B}$ equation and the fKdV equation, with the viscous effect being taken into account crudely, showed good 
agreement with the experimental data. Later, Teng \& Wu (1997) carried out a similar experimental study with two identical vertical struts along the channel sidewalls. The shape and size of the cross-section of the struts were the same as those of the two-dimensional cylinder in the experiments of Lee et al. (1989). The half-width of the channel was $5 \mathrm{~cm}$, resulting in a blockage coefficient of 0.132 .

It is interesting to note that the detailed geometry of the elliptic cylinder used in the present numerical calculations is different from that of the arched cylinder used in the aforementioned experiments, but as long as the blockage coefficient is kept the same, the amplitudes of solitary waves predicted by the present NS method would agree well with the experiments. However, as will be discussed in the next section, the body shape does play a significant role in the generation of solitons in a viscous flow.

It should be noted that the physical models used in the experiments of Lee et al. (1989) and Teng \& Wu (1997) were very small and the Reynolds numbers were of the order of $10^{4}$. The viscous effect was significant in these experiments. Therefore, an empirically determined effective bump height of $0.8 \mathrm{~cm}$ instead of the actual bump height of $0.65 \mathrm{~cm}$ was used in their inviscid numerical calculations of the $\mathrm{g}-\mathrm{B}$ equation and the $\mathrm{fKdV}$ equation to crudely account for the viscous effect. Otherwise, the comparison between the theoretical and experimental results would be poor. However, for experiments of high Reynolds numbers, say $10^{6}$, the overall viscous effect on the generation of solitary waves may be insignificant. The predictions by the inviscid theoretical models would be comparable with the experiments. The present NS method has been used to simulate the experiments of Lee et al. (1989) by Zhang \& Chwang (1996). Using the exact geometry as in the experiments, the present NS method accurately predicted all the characteristics of solitary waves observed in the experiments. Further study on solitary waves generated by three-dimensional bodies such as surface ships is of interest, and can be carried out by the present method.

\subsection{The effect of body shape on solitary-wave generation}

Different studies based on the approximate inviscid flow theory (Akylas 1984; Grimshaw \& Smyth 1986; Smyth 1987) have shown that the generation of solitary waves depends on the total force only, and not on the precise detail of the excitation. In the present study, the shape effect on the solitary-wave generation is investigated by more accurate, fully nonlinear models based on viscous and inviscid flow theories, i.e. the NS and the Euler equations. By dropping the viscous terms, the governing NS equations reduce to the Euler equations. The boundary conditions described above must be modified accordingly. For instance, the no-slip condition applied on the body surface should be replaced by the no-penetration condition.

The calculation cases 6, 7, 1, 8, and 9 (group 2) are designed to investigate the shape effect on the solitary-wave generation. All the five elliptic cylinders have the same cross-sectional area of $3 \mathrm{~cm}^{2}$, meaning the same forcing intensity. The major-to-minor axis ratios of the five elliptic cylinders are 2, 4, 6, 8, and 10, respectively, and the water depth varies in such a way that the same blockage coefficient $C_{b}=0.20$ is maintained for the five calculation cases (see table 1). Wave elevations obtained from the Euler solutions for the five cases at time $t=120$ are shown in figure 9. It can be seen from the figure that the upstream-advancing solitary waves are essentially the same for all five cases. For the cases of major-to-minor axis ratio greater than 4 , the difference in depressed water regions and trailing waves are still minor. Noticeable differences are seen only for case $6(a / b=2)$, in which the depressed water region becomes shorter with a higher water level and the amplitudes of trailing waves become smaller. The results indicate that, in inviscid flow, the shape of a body has no effect on the 


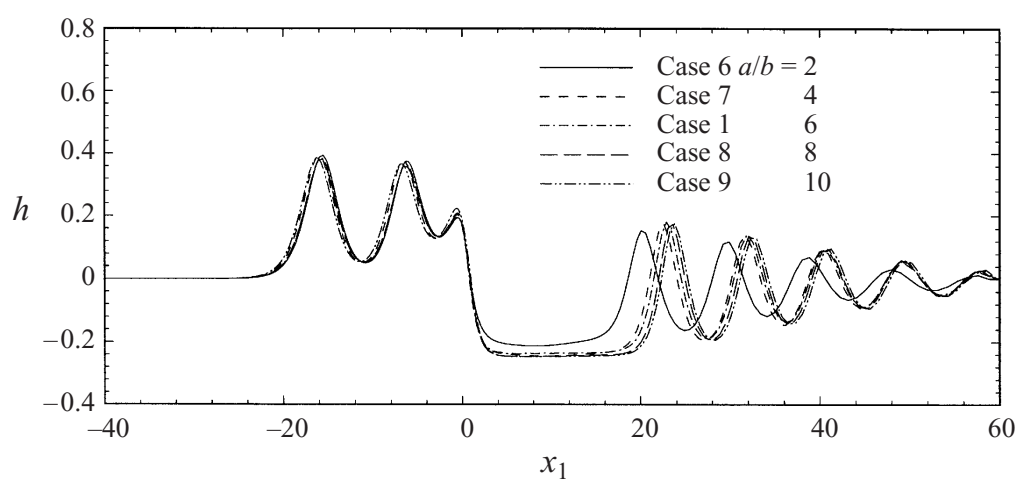

FIGURE 9. Free-surface elevation $h$ at time $t=120$ for cylinders of different shape with the same blockage coefficient $C_{b}=0.2$ (Euler solutions).

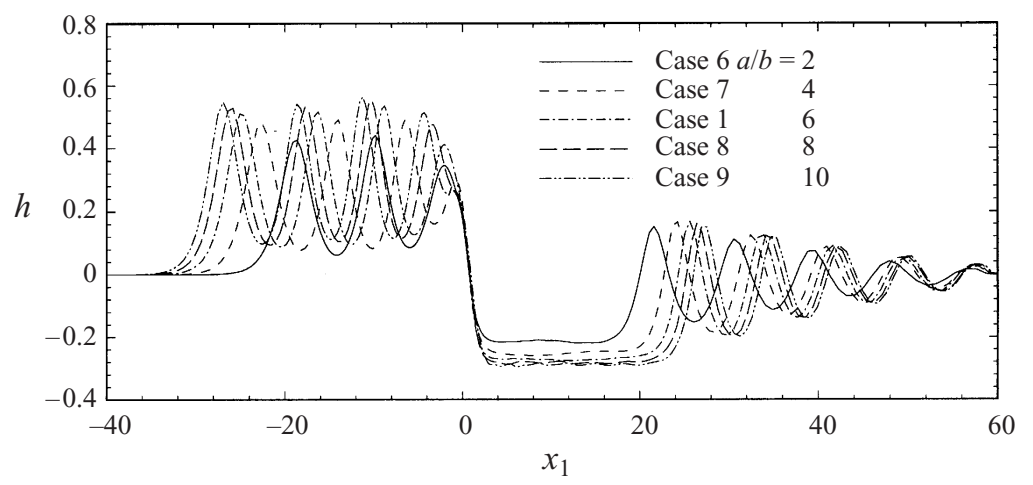

FIGURE 10. Free-surface elevation $h$ at time $t=120$ for cylinders of different shape with the same blockage coefficient $C_{b}=0.2$ (Navier-Stokes solutions).

generation of upstream-advancing solitary waves, but has an effect on the depressed water region and trailing waves when the length of the body is sufficiently short.

Computations with the NS equations are carried out for the same five bodies to investigate the shape effect on the wave generation in viscous flow. Similar plots to those in figures 7 and 8 are given in figures 10 and 11 to display the effect of body shape, i.e. the major-to-minor axis ratio $a / b$, on the solitary-wave generation. Similar trends to those shown in figures 7 and 8 for variations of amplitude and period with $C_{b}$ are now seen in figures 10 and 11 for variations of amplitude and period with the major-to-minor axis ratio $a / b$. In figure 12 , the NS and Euler solutions are shown together for each case separately. The effect of viscosity on wave generation can be seen distinctly. As can be seen, the longer the body, the stronger the viscous effect. The effect stems from the viscous boundary layer in the immediate neighbourhood of the solid-body surface (see figures 4 and 5). The presence of a viscous boundary layer around the body may be sought to increase the effective disturbance caused by the body. If a shape change results in an increase in the affected region of its boundary layer, the influence on the flow by the body would increase. For elliptic cylinders of constant cross-sectional area, the larger the major-to-minor axis ratio, the bigger the area of the solid surface, and so the stronger the viscous effect.

Flow behaviour in a viscous fluid is highly dependent on the Reynolds number. Tang, Patel \& Landweber (1990) investigated the viscous damping of a free-running 


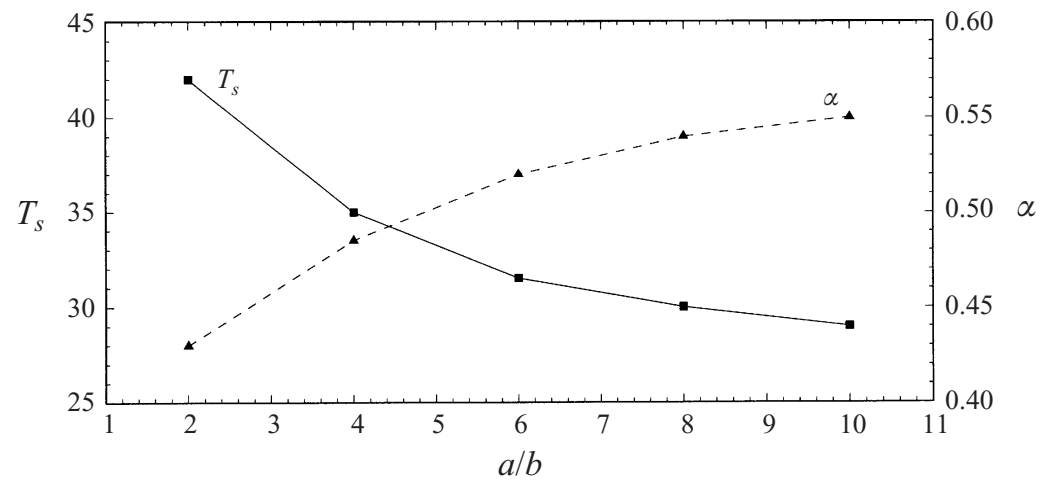

FIGURE 11. Wave amplitude $\alpha$ and period $T_{S}$ versus the major-to-minor axis ratio $a / b$.
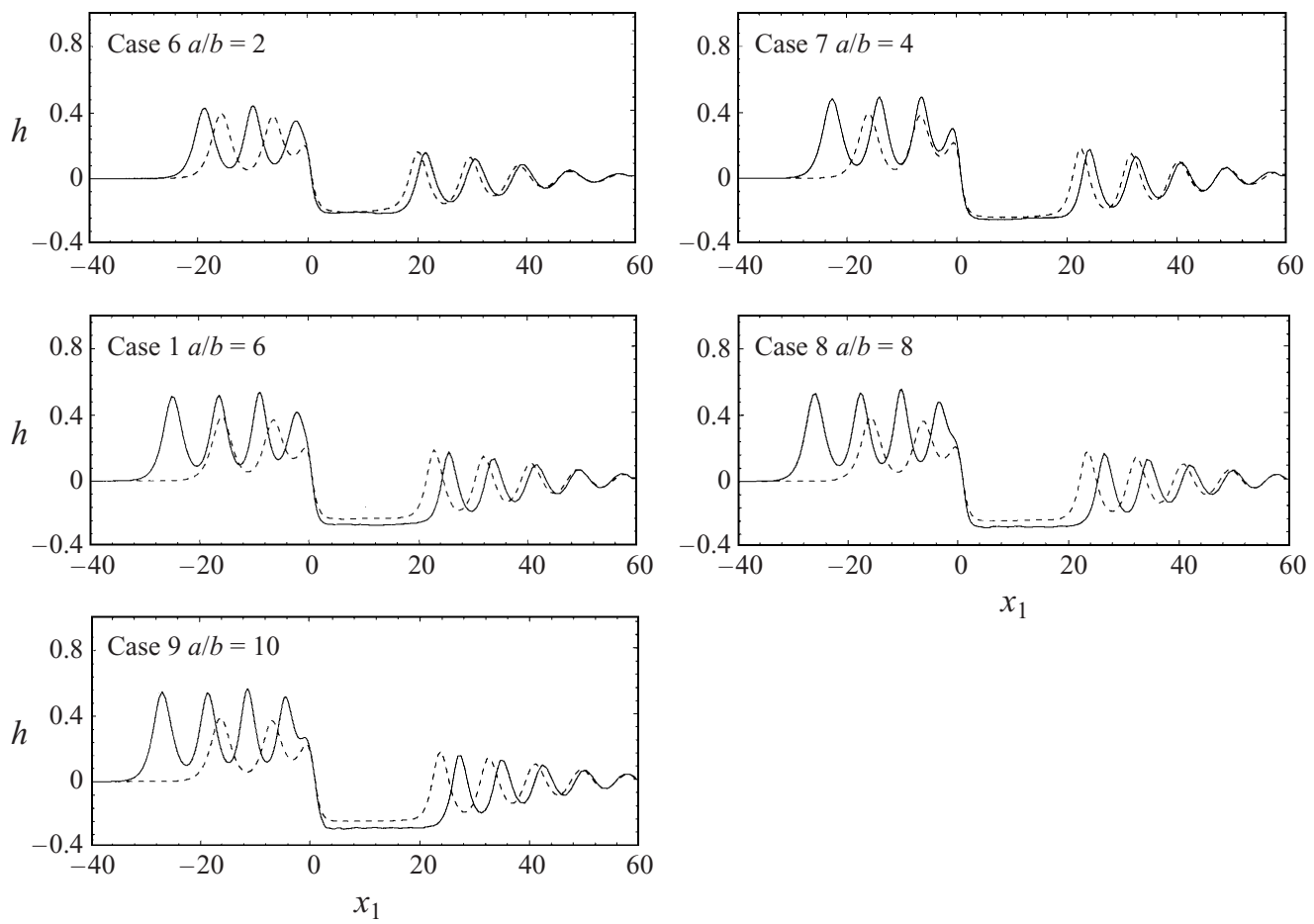

FiguRE 12. Comparisons of free-surface elevation $h$ at time $t=120$ for cylinders of different shape with the same blockage coefficient $C_{b}=0.2$. - NS solution; ---, Euler solution.

solitary wave in a two-dimensional shallow water channel by numerically solving the NS equations for three different values of the Reynolds number, $R e=50,500$, and 50000 . They found that the viscous damping of the wave decreases with increasing Reynolds number. The viscous damping comes from both the bottom of the channel and the free surface, with that from the former being much stronger. In the present calculations, the Reynolds number is of the order of $10^{4}$ (see table 1). Under these circumstances, the viscous effect on the solitary-wave generation owing to a change of the body shape comes mainly from the viscous boundary layers around the body and at the bottom. 
The viscous effect on solitary waves generated by underwater moving objets depends on factors such as the flow separation, formation of vortices in the boundary layer and in the wake of the body, and the interaction between waves and viscosity. It is well established that the boundary layer and the wake can influence the resulting wave structure through the displacement thickness effect, and the wave structure, in turn, can influence the boundary layer through wave elevation and the wave-induced pressure gradient.

\subsection{A preliminary discussion on the limit of the resonant regime}

The above discussion on the generation of solitary waves by underwater moving objects is concentrated entirely on the critical case in which the Froude number is exactly unity. Experiments, numerical simulations and theoretical analyses all indicate the existence of a transcritical speed range in which an upstream train of solitary waves of uniform amplitude is observed (Huang et al. 1982; Ertekin et al. 1984; Miles 1986; Grimshaw \& Smyth 1986; Wu 1987; Melville \& Helfrich 1987; Lee et al. 1989). For single-layer flows, Miles (1986) predicted the lower and upper critical Froude numbers $\left(F_{l}, F_{u}\right)$ analytically via the Rayleigh asymptotic process. They are $F_{l}^{2}=1-\left(\frac{9}{2} \varepsilon\right)^{2 / 3}$ and $F_{u}^{2}=1+\left(\frac{9}{4} \varepsilon\right)^{2 / 3}$. Here, $\varepsilon$ is the blockage coefficient which measures the strength of the forcing. Grimshaw \& Smyth (1986, hereinafter referred to as GS) described the flow of a continuously stratified fluid over topography for both the resonant and non-resonant cases by the fKdV equation. They showed that the resonant limits are defined by $\Delta_{-}<\Delta<\Delta_{+}$. Here $\Delta$ is the detuning parameter, and $\Delta_{-}$and $\Delta_{+}$are the lower and upper limits which are scaled differently with the forcing parameters for broad and narrow obstacles. In order to make comparisons easily, Melville \& Helfrich (1987) extended Miles' (1986) results to two-layer flows (described by the fKdV equation), and then expressed both Miles' (1986) and GS' (1986) results in terms of the Froude number and the effective blockage coefficient $\gamma$ (see their equations (5.2), (5.3), (5.4) for more details). The upper bounds were shown in their figure 9. It is seen from this figure that values of the upper critical Froude number predicted by different theories are different. For instance, when $\gamma=0.0625$, the $\left(F_{l}, F_{u}\right)$ predicted by Miles and GS for a narrow obstacle and GS for a broad obstacle are $(0.72,1.17),(0.86,1.28)$ and $(0.9,1.21)$, respectively. $F_{l}$ shows a $19 \%$ difference between Miles' prediction and GS' prediction for a narrow obstacle, and a $25 \%$ difference between Miles' prediction and GS' prediction for a broad obstacle, while $F_{u}$ shows a $9 \%$ and $3 \%$ difference, repectively.

It is of interest to examine the existence of a transcritical regime for the generation of solitary waves by the present fully nonlinear model. A number of computations for case 1 with the Froude number increasing from 0.85 to 1.05 are carried out for a preliminary investigation. Figure 13 shows the qualitatively different solutions obtained for different Froude numbers with the forcing strength and the blockage coefficient being fixed. It is seen that as the Froude number increases, the solution goes from upstream undular bores to solitary waves, and then back to undular bores again. More numerical runs at Froude numbers between 0.9 and 1.05 with an interval of 0.01 are carried out and the results seem to indicate that $F_{l}$ and $F_{u}$ are 0.95 and 1.03 , respectively.

Tomasson \& Melville (1991) found an upstream front-like solution in the shallowwater model including the cubic nonlinearity. However, when the Froude number is within the range of $0.85-1.05$, no upstream front-like solutions appear in the present fully nonlinear model. 


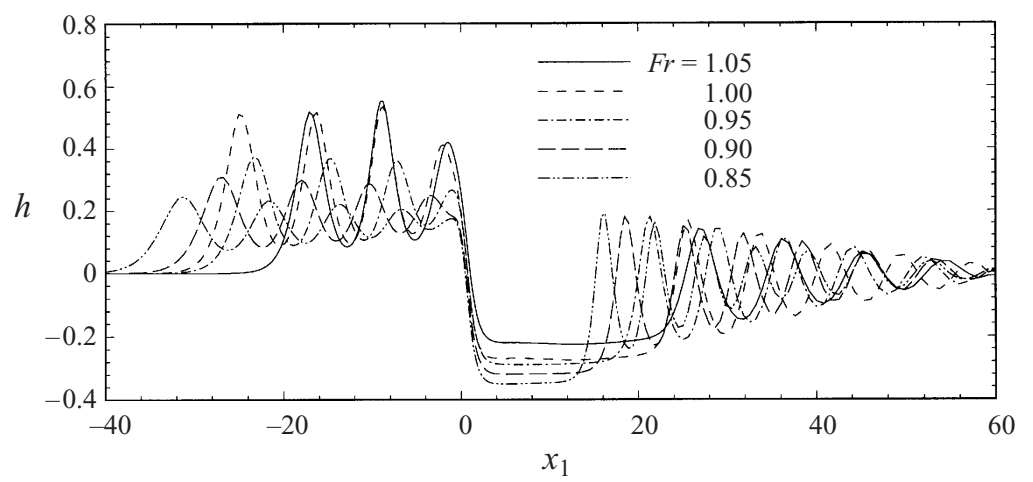

FIGURE 13. Free-surface elevation $h$ at time $t=120$ from computational results of case 1 (NS solution; $a / b=6$ ) at Froude numbers from 0.85 to $1.05 . C_{b}=0.2$.

\section{Conclusions}

A series of calculations for nine elliptic cylinders of the same cross-sectional area but of different shape moving steadily at critical speed in shallow water channels of different water depths is carried out to study the blockage effect and the shape effect on the generation of solitary waves. The overall features of the generated solitary waves are accurately simulated by the present numerical method of NS equations. The relation between the period of solitary-wave generation and its amplitude, calculated by the present numerical method matches well with Wu's (1987) theoretical formula based on the mass, momentum and energy considerations of the fKdV equation. The relation between the amplitude and celerity of solitary waves calculated by the present numerical method is also in good agreement with those of two theoretical formulae, one given by Rayleigh and the other derived from KdV model. Furthermore, the detailed information of the flow, such as the pressure distribution, velocity and vorticity fields, is available from the present method.

The present study confirms the findings of experiments that the blockage coefficient has a significant effect on the solitary-wave generation. The wave amplitude increases while the period of generation decreases as the blockage coefficient increases. The effect of a body shape on the solitary-wave generation is investigated by using both the NS and Euler equations. It is found that, in inviscid flow, the shape of a body under the free surface has no effect on the generation of upstream-advancing solitary waves, but has an effect on the depressed water region and trailing waves when the body length is sufficiently short (body-length/water-depth ratio $2 a / h_{0}<0.8$ in the present study). While in viscous flow, the shape of a body under the free surface has a significant effect on the solitary-wave generation through viscous effect in the boundary layer of the body. In general, if a change in shape results in increasing the area of the body surface, the viscous effect will be enhanced, and so will the disturbance forcing. Therefore, the amplitude of solitary waves increases while the period of generation decreases. For two-dimensional cylinders of elliptic cross-section of the same area, the thinner (larger major-to-minor axis ratio) the cross-section is, the larger the amplitude and frequency of the solitary waves will be.

This research was jointly sponsored by the Hong Kong Research Grants Council under Grant nos. HKU 304/95E and HKU 568/96E and by the University of Hong Kong under CRCG Grant no. 337/064/0032. 


\section{REFERENCES}

Akylas, T. R. 1984 On the excitation of long nonlinear water waves by a moving pressure distribution. J. Fluid Mech. 141, 455-466.

BAI, K. J. \& Kim, Y. H. 1989 Numerical computations for a nonlinear free surface flow problem. In Proc. Fifth Intl Conf. on Numerical Ship Hydrodyn. Hiroshima, Japan, pp. 305-320.

CAO, Y. \& BECK, R. F. 1993 Numerical computations of two-dimensional solitary waves generated by moving disturbances. International Journal for Numerical Methods in Fluids, 17, 905-920.

Chan, R. K. C. \& Street, R. L. 1970 A computer study of finite-amplitude water waves. J. Comput. Phys. 6, 68-94.

Chang, J. H. \& TANG, C. J. 1993 Viscous effects on nonlinear water waves generated by a submerged body in critical motion. In Proc. 17th Natl Conf. on Theoret. Appl. Mech. Taipei, Taiwan, pp. $35-42$.

Chen, X. N. \& Sharma, S. D. 1994 Nonlinear theory of asymmetric motion of a slender ship in a shallow channel. In Proc. of 20th Symp. on Naval Hydrodyn. (ed. E. P. Rood), pp. 386-407. Santa Barbara, CA.

Chen, X. N. \& Sharma, S. D. 1995 A slender ship moving at a near-critical speed in a shallow channel. J. Fluid Mech. 291, 263-285.

Chor, H. S., BaI, K. J., Kim, J. W. \& Cho, H. 1991 Nonlinear free surface waves due to a ship moving near the critical speed in a shallow water. In Proc. 18th Symp. on Naval Hydrodyn. pp. 173-190. National Academy Press, Washington DC.

Cole, S. L. 1985 Transient waves produced by flow past a bump. Wave Motion 7, 579-587.

Ertekin, R. C., Webster, W. C. \& Wehausen, J. V. 1984 Ship-generated solitons. In Proc. 15th Symp. on Naval Hydrodyn. Hamburg, pp. 347-364.

Ertekin, R. C., Webster, W. C. \& Wehausen, J. V. 1986 Waves caused by a moving disturbance in a shallow channel of finite width. J. Fluid Mech. 169, 275-292.

Ertekin, R. C. \& Qian, Z. M. 1986 Numerical grid generation and upstream waves for ships moving in restricted waters. In Proc. Fifth Intl Conf. on Numerical Ship Hydrodyn. Hiroshima, Japan, pp. 421-437.

Ertekin, R. C., Qian, Z. M. \& Wehausen, J. V. 1990 Upstream solitons and wave resistance. In Engineering Science, Fluid Dynamics, pp. 29-43. World Scientific.

Farmer, J., Martinelli, L. \& Jameson, A. 1994 Fast multigrid method for solving incompressible hydrodynamic problems with free surfaces. AIAA J. 32, 1175-1182.

Ferziger, J. H. \& Peric, M. 1996 Computational methods for Fluid Dynamics. Springer.

GrafF, W. 1962 Untersuchungen über die Ausbildung des Wellenwiderstandes im Bereich der Stauwellengeschwindigkeit in flachem, seitlich beschränktem Farhwasser. Schifftechnik 9, 110 122.

Grimshaw, R. H. J. \& Smyth, N. 1986 Resonant flow of a stratified fluid over topography. J. Fluid Mech. 169, 429-464.

Harlow, F. H. \& Welch, J. E. 1965 Numerical calculation of time-dependent viscous incompressible flow of fluid with free surface. Phys. Fluids 8, 2182-2189.

HINATSU, M. 1992 Numerical simulation of unsteady viscous nonlinear waves using moving grid system fitted on a free surface. J. Kansai Soc. $N$ A, Japan, 217, 1-11.

Huang, D. B., Sibul, O. J., Webster, W. C., Wehausen, J. V., Wu, D. M. \& Wu, T. Y. 1982 Ships moving in the transcritical range. In Proc. Conf. on Behavior of Ships in Restricted Waters, vol. 2, Varna, Bulgarian Ship Hydrodynamics Center, pp. 26-1-26-10.

Katsis, C. \& Akylas, T. R. 1987 On the excitation of long nonlinear water waves by a moving pressure distribution. Part 2. Three-dimensional effects. J. Fluid Mech. 177, 49-65.

LeE, S. J., Yates, G. T. \& WU, T. Y. 1989 Experiments and analyses of upstream-advancing solitary waves generated by moving disturbances. J. Fluid Mech. 199, 569-593.

LEONARD, B. P. 1979 A stable and accurate convective modeling procedure based on quadratic upstream interpolation. Comput. Meth. Appl. Mech. Engng 19, 59-98.

MeI, C. C. 1986 Radiation of solitons by slender bodies advancing in a shallow channel. J. Fluid Mech. 162, 53-67.

MeI, C. C. \& CHOI, H. S. 1987 Forces on a slender ship advancing near the critical speed in a wide canal. J. Fluid Mech. 179, 59-76. 
Melville, W. K. \& Helfrich, K. R. 1987 Transcritical two-layer flow over topography. J. Fluid Mech. 178, 31-52.

Miles, J. W. 1986 Stationary, transcritical channel flow. J. Fluid Mech. 162, 489-499.

Miyata, H. \& Nishimura, S. 1985 Finite-difference simulation of nonlinear ship waves. J. Fluid Mech. 157, 327-357.

Newman, J. N. 1980 Marine Hydrodynamics. The MIT press, Cambridge, Massachusetts.

Pedersen G. 1988 Three-dimensional wave patterns generated by moving disturbances at transcritical speeds. J. Fluid Mech. 196, 39-63.

Protopopov, B. E. 1991 Numerical investigation of soliton generation by a moving region of free surface pressure. Intl Ser. Numer. Maths 99, 347-355.

Schmidt-Stiebitz, H. 1966 Die Abhängigkeit des Schiffswiderstandes von flachwasserbedingten Umströmungs-und Wasserspiegelveränderungen. Schiff und Hofen 18, 381-395.

Syмтн, N. 1987 Modulation theory solution for resonant flow over topography. Proc. R. Soc. Lond. A 409, 79-97.

Sun, M. G. 1985 The evolution of waves created by a ship in a shallow canal. In The 60th Anniv. Volume, Zhongshan University, Mechanics Essays, pp. 17-25. Guangzhou, China, (in Chinese).

Tang, C. J., Patel, V. C. \& Landweber, L. 1990 Viscous effects on propagation and reflection of solitary waves in shallow channels. J. Comput. Phys. 88, 86-113.

Teng, M. H. \& WU, T. Y. 1992 Nonlinear water waves in channels of arbitrary shape. J. Fluid Mech. 242, 211-233.

Teng, M. H. \& Wu, T. Y. 1997 Nonlinear shallow water waves generated by submerged moving slender bodies: an experimental study. In Proc. 7th Intl Offshore and Polar Engng Conf. vol. 3, pp. 313-317.

Thews, J. G. \& LandweBer, L. 1935 The influence of shallow water on the resistance of a cruiser model. US Experimental Model Basin Report 408, Washington, DC, Navy Yard.

Tomasson, G. G. \& Melville, W. K. 1991 Flow past a constriction in a channel: a modal description. J. Fluid Mech. 232, 21-45.

WU, D. M. \& WU, T. Y. 1982 Three-dimensional nonlinear long waves due to moving surface pressure. In Proc. 14th Symp. on Naval Hydrodyn, pp. 103-125. Washington DC.

Wu, T. Y. 1981 Long waves in ocean and coastal waters. J. Engng Mech. Div. ASCE 107, 501-522.

$\mathrm{Wu}$, T. Y. 1987 Generation of upstream advancing solitons by moving disturbances. J. Fluid Mech. 184, 75-99.

Yeung, R. W. \& Vaidhyanathan, M. 1992 Nonlinear interaction of water waves with submerged obstacles. Intl J. Numer. Meth. Fluids 14, 1111-1130.

Zhang, D. H. \& Chwang, A. T. 1996 Numerical study of nonlinear shallow water waves produced by a submerged moving disturbance in viscous flow. Phys. Fluids 8, 147-155. 